\title{
ON THE LINEAR TRANSFORMATIONS OF A QUADRATIC FORM INTO ITSELF*
}

BY

\section{PERCEY F. SMITH}

The problem of the determination $\dagger$ of all linear transformations possessing an invariant quadratic form, is well known to be classic. It enjoyed the attention of Euler, Cayley and Hermite, and reached a certain stage of completeness in the memoirs of Frobenius, $\ddagger$ Voss, $§$ Lindemans $\|$ and Loewr. $\uparrow$ The investigations of CAYLEY and Hermite were confined to the general transformation, Frobenius then determined all proper transformations, and finally the problem was completely solved by LindeManN and LoEwy, and simplified by Voss.

The present paper attacks the problem from an altogether different point, the fundamental idea being that of building up any such transformation from simple elements. The primary transformation is taken to be central reflection in the quadratic locus defined by setting the given form equal to zero. This transformation is otherwise called in three dimensions, point-plane reflection,point and plane being pole and polar plane with respect to the fundamental quadric. In this way, every linear transformation of the desired form is found to be a product of central reflections. The maximum number necessary for the most general case is the number of variables. Voss, in the first memoir cited, proved this theorem for the general transformation, assuming the latter given by the equations of CAYLEY. In the present paper, however, the theorem is derived synthetically, and from this the analytic form of the equations of transformation is deduced.

\footnotetext{
* Presented to the Society December 29, 1903. Received for publication, July 2, 1r04.

$\dagger$ The results of $\S \S 1,2$ were communicated to the American Mathematical Society in December, 1901.

$\ddagger$ Frobenivs, Ueber linear substitutionen und bilinear Formen, Cr ell e, vol. 84 (1878), pp. 1-63.

§Voss, Zur theorie der orthogonalen substitutionen; Mathematische Annalen, vol. 13 (1878), pp. 320-374; Ueber die cogrediente Transformation der bilinearen formen in sich selbst, Münchener Berichte (1896), pp. 1-23.

|| Lindemann, Vorlesungen über Geometrie, vol. 2 (1891), p. 356 ; Ueber die linearen Transformationen einer quadratischen Mannigfaltigkeit in sich, Münchener Berichte (1896), pp. 31-66.

TLow y, Ueber die Transformationen einer quadratischen Form in sich selbst, Le op. Nova A cta, Halle, vol. 65 (1896), pp. 1-66.
} 
The theory of central reflections developed in $\S 1$ is so simple, and the analytic representation derived therefrom so direct that the present discussion presents a complete solution of the problem which may be regarded as elementary. Furthermore, the equations (24) found in explicit form for every such transformation have not been given elsewhere, as far as the author's knowledge goes.

In $\S \S 5,6$ are given some applications, and in $\S 7$ the solution of the corresponding problem for the alternating bilinear form. The results of the first six sections apply, of course, to the symmetrical bilinear form.

Let

\section{\$1. The theory of central reflections.}

be the given quadratic form. The quadratic locus $f(x)=0$ in linear space $R_{n-1}$ of $n-1$ dimensions may be called after Cayley the "absolute." Any group of $r$ independent sets of coördinates

$$
\left(a_{1}^{(1)}, a_{2}^{(1)}, \ldots, a_{n}^{(1)}\right), \ldots,\left(a_{1}^{(r)}, a_{2}^{(r)}, \ldots, a_{n}^{(r)}\right),
$$

determine a linear manifold $M_{n-r-1}$ of $n-r-1$ dimensions, the coördinates $x$ of any point of which are linearly derived from the $a$ 's:

$$
x_{i}=\lambda^{(1)} a_{i}^{(1)}+\lambda^{(2)} a_{i}^{(2)}+\cdots+\lambda^{(r)} a_{i}^{(r)} \quad(i=1,2, \cdots, n) .
$$

The involutory transformation which forms the basis of the discussion is the "central reflection" of Voss (loc. cit.), or the point-plane reflection, as it is often called, point and plane standing in the relation of pole and polar with respect to $f(x)=0$. I shall denote by $\{a\}$ the reflection in the point $a$ and its polar plane, and by $x\{a\} x^{\prime}$ the fact of $x$ transforming into $x^{\prime}$, from which also, since the transformation is involutory, will follow $x^{\prime}\{a\} x$. From the definition of the transformation $x^{\prime}, x$ and $a$ are collinear, hence $x^{\prime}=x+\lambda a$, and since by hypothesis, $f\left(x^{\prime}\right) \equiv f(x)$, we find for the equations of $\{a\}$

$$
x^{\prime}=x-\frac{2 f(x, a)}{f(a)} a, \quad \text { or } \quad x=x^{\prime}-\frac{2 f\left(x^{\prime}, a\right)}{f(a)} a,
$$

where, of course, $f(a) \neq 0$ (and may be taken equal to 1 when desired), because the center a must not lie on the absolute.

Evidently the center $a$ and each point in the polar $M_{n-2}$ of $a$, viz., $f(x, a)$ $=0$ are invariant under $\{a\}$.

The product $\{a b\}$ of two reflections $\{a\}$ and $\{b\}$ comes out as

$$
x^{\prime}=x-\frac{2 f(x, a)}{f(a)} a-\frac{2 f(x, b)}{f(b)} b+\frac{4 f(x, a) f(a, b)}{f(a) f(b)} b .
$$

This is symmetrical in $a$ and $b$ when and oniy when $f(a, b)=0$, i. e., we have the result : 
Two central reflections $\{\mathbf{a}\}$ and $\{\mathbf{b}\}$ are commutative, when and only when $a$ and $b$ are conjugate points with respect to the absolute.*

The important question is now this: When is $\{\mathbf{a b}\} \equiv\{\mathbf{c d}\}$ ?

A necessary condition is expressed in

Theorem 1. If $\left\{\mathbf{a}^{(1)} \mathbf{a}^{(2)} \mathbf{a}^{(3)} \cdots \mathbf{a}^{(r)}\right\} \equiv\left\{\mathbf{b}^{(1)} \mathbf{b}^{(2)} \mathbf{b}^{(3)} \cdots \mathbf{b}^{(r)}\right\}$, then each of the centers $b$ is numerically derived from the centers $a^{(1)}, a^{(2)}, \ldots, a^{(r)} \cdot \dagger$

Take $r=2$. Then from (3), $\{a b\} \equiv\{c d\}$ gives as a necessary and sufficient condition

$$
\begin{aligned}
\frac{f(x, a)}{f(a)} a+\left(\frac{f(x, b)}{f(b)}-\right. & \left.\frac{2 f(x, a) f(a, b)}{f(a) f(b)}\right) b \\
& \equiv \frac{f(x, c)}{f(c)} c+\left(\frac{f(x, d)}{f(d)}-\frac{2 f(x, c) f(c, d)}{f(c) f^{\prime}(d)}\right) d
\end{aligned}
$$

for every value of $x$. If $c \neq=a$, take $f(x, c)=0$, and $f(x, a) \neq 0$. Then $d$ is numerically derived from $a$ and $b$. Similarly for $c$.

The following proof involving the principles of Grassmans's Ausdehnungslehre $\ddagger$ is general and very direct. Regarding $a, b, c, d$ as extensive magnitudes or complex quantities, and the coefficients in (4) as scalars, we notice that the coefficient of $b$ is of the same form as that of $a$ diminished by a multiple of the latter. Now write down ( 4 ) with $x$ replaced by $x^{\prime}$, and multiply the corresponding members of the two equations together using the "combinatorial law" $a b=-b a, c d=-d c$; then, taking $f(a)=f(b)=f(c)=f(d)=1$, we get easily

$$
\left|\begin{array}{ll}
f(x, a) & f(x, b) \\
f\left(x^{\prime}, a\right) & f\left(x^{\prime}, b\right)
\end{array}\right| a b \equiv\left|\begin{array}{ll}
f(x, c) & f(x, d) \\
f\left(x^{\prime}, c\right) & f\left(x^{\prime}, d\right)
\end{array}\right| c d,
$$

in which the scalar coefficients are determinants. In this form the theorem follows at once, and as the method is in no wise different for the general case, the truth of the proposition is established.

It is now necessary to add only the following theorem, after which a complete theory of central reflections may be developed by synthesis.

Theorem 2. The product $\{\mathbf{a b}\}$ of two central reflections may be resolved in $\infty^{1}$ ways into $\{\mathrm{cd}\}$, the centers $c$ and $d$ lying on the manifold $M_{1}$ derived from $a$ and $b$. Either $c$ or $d$ may be chosen arbitrarily on this line. The other is then uniquely determined.

For if $f(a)=1, f(b)=1$, and $\lambda^{2}+\mu^{2}+2 \lambda \mu f(a, b)=1$, then $\{\mathbf{a b}\} \equiv\{\mathrm{cd}\}$ if $c=\lambda a+\mu b$, and $d=-\mu a+[\lambda+2 \mu f(a, b)] b$, as direct substitution in

* Voss, loc. cit., p. 345.

† We assume $r \ngtr n$, and $a_{1}, a_{2}, \cdots, a_{r}$ independent.

$\ddagger$ Ausdehnungslehre von 1862 , p. 42 . 
(4) will show.* Furthermore, we cannot write $\{\mathbf{a b}\} \equiv\{\mathbf{c d}\} \equiv\left\{\mathbf{c d}^{\prime}\right\}$ unless $\{d\} \equiv\left\{\mathbf{d}^{\prime}\right\}$. For $\{\operatorname{ccd}\}$ would in this case be identical with $\left\{\operatorname{ccd}^{\prime}\right\}$, and since $\{c c\}$ is the identical transformation, we must also have $\{d\} \equiv\left\{\mathbf{d}^{\prime}\right\}$.

The invariant configuration of $\{a b\}$ is made up of the points of intersection of the line $a b$ with the absolute, and every point of the $M_{n-3}$ common to the two polar manifolds $M_{n-2}$ of $a$ and $b$. $\dagger$

It is to be noted also that $\lambda a+\mu b$ lies upon the absolute when

$$
\lambda^{2}+\mu^{2}+2 \lambda \mu f(a, b)=0,
$$

which is the condition of coincidence of $c$ and $d$, i. e., in the resolution of $\{a b\}$ the express hypothesis made in (2) that a center shall not lie upon the absolute is equivalent to coincidence of $c$ and $d$.

Pass on now to $r=3$, and the proof of

Theоnem 3. The product of three central reflections $\{$ abc $\}$ may always be resolved in $\infty^{3}$ ways into $\{$ def $\}$. The first center is any point in the plane of $a, b, c$; but the line joining $e$ and $f$ is then uniquely determined, though either $e$ or $f$ may be chosen at will upon this line.

Given, then, the three centers $a, b, c$, and any center $d$ in their plane. If the point $b^{\prime}$ of intersection of $a d$ and $b c$ is not on the absolute, then by theorem $2,\{\mathbf{a b c}\} \equiv\left\{\mathbf{a b}^{\prime} \mathbf{c}^{\prime}\right\} \equiv\{$ def $\}$. Suppose, however, $f\left(b^{\prime}\right)=0$, and let $a^{\prime}$ be any center on $a b$, so that we may write $\{\mathbf{a b}\} \equiv\left\{\mathbf{a}^{\prime} \mathbf{b}^{\prime}\right\}$. Then the lines $d a^{\prime}$ and $c b^{\prime}$ are projectively related, and accordingly their point of intersection $b^{\prime \prime}$ describes a conic containing $d$ and $c$. Since $d$ and $c$ are not on the absolute, this conic cannot be the intersection of the plane of centers with the absolute, and $b^{\prime \prime}$ may be assumed a center. Hence we now have $\{\mathbf{a b c}\} \equiv\left\{\mathbf{a}^{\prime} \mathbf{b}^{\prime} \mathbf{c}\right\} \equiv\left\{\mathbf{a}^{\prime} \mathbf{b}^{\prime \prime} \mathbf{c}^{\prime}\right\} \equiv\{$ def $\}$, and the first part of theorem 3 is proved.

For the rest, if $($ def $) \equiv\left(d e^{\prime} f^{\prime}\right)$, then $(e f) \equiv\left(e^{\prime} f^{\prime}\right)$ and the second part follows at once from theorem 2 .

It appears, therefore, that the transformation \{abc $\}$ establishes in the plane of centers a point-line relation between the first center $d$ and the line ef upon which the remaining two must then lie.

This relation is a correlation. For if $f$ is any center on ef', then $\{$ def $\} \equiv\left\{\mathrm{d}_{1} \mathrm{e}_{1} \mathrm{f}_{1}\right\}$; and if $d e$ and $d_{1} e_{1}$ intersect in $d^{\prime}$, we may write $\left\{\mathrm{d}^{\prime} \mathrm{e}^{\prime} \mathrm{f}\right\} \equiv\left\{\mathrm{d}^{\prime} \mathrm{e}_{1}^{\prime} \mathbf{f}_{1}\right\}$. Hence $e^{\prime}$ and $e_{1}^{\prime}$ are on $e f$, and $e^{\prime}$ and $d^{\prime}$ coincide with $e$ and $d$ respectively. Therefore as $f$ in $\{$ def $\}$ moves along $e f$, the corresponding line turns around $d$. Hence the transformation $\{$ abc $\}$ establishes a duality $\{\Gamma\}$ in the plane of centers, such that the second and third centers lie upon the line that corresponds under $\{\boldsymbol{\Gamma}\}$ to the first center. Furthermore, since

* The equations simply indicate that $a, b$ and $c, d$ are corresponding points of a projectivity on the line of centers and with fixed points upon the absolute.

$\dagger$ The line joining $a$ and $b$ touches the absolute when $f(a) f(b)=[f(a, b)]^{2}$. 
$\{$ def $\} \equiv\left\{\right.$ ed'f $^{\prime} \equiv\left\{\mathbf{e f d}^{\prime \prime}\right\}, f$ lies on the correlate of $e$, and finally, as the preceding shows that a point and its line under $\{\Gamma\}$ are united only when the point is on the absolute, we have

THEOREM 4. The transformation \{abc $\}$ establishes in the plane of centers a duality which is characteristic of the transformation. In any resolution of $\{\mathbf{a b c}\}$, the line corresponding in this duality to any center contains the following centers of the product. Coincidence of point and line under this duality occurs when and only when the point is on the absolute.

The generalization of this theorem is at once accomplished by complete induction. Assume, then, that theorems 3 and 4 hold for the product of any $p$ central reflections $p<n$. Write the transformation

$$
\left\{\mathbf{T}_{p+1}\right\} \equiv\left\{\mathbf{a}^{(1)} \mathbf{a}^{(2)} \cdots \mathbf{a}^{(p)} \mathbf{a}^{(p+1)}\right\} \equiv\left\{\mathbf{a}^{(1)} \mathbf{T}_{p}\right\}
$$

Let $b^{(1)}$ be any point in the $M_{p}$ numerically derived from $a^{(1)}, a^{(2)}, \ldots, a^{(p+1)}$, and suppose the line $a^{(1)} b^{(1)}$ intersects the locus of centers of $\left\{\mathbf{T}_{p}\right\}$ in $a^{(0)}$. Considerations analogous to the preceding permit us to assume $a^{(0)}$ not on the absolute, and we may by hypothesis write $\left\{\mathbf{T}_{p}\right\} \equiv\left\{\mathbf{a}^{(0)} \mathbf{T}_{p-1}\right\}$. Since also $\left\{\mathbf{a}^{(1)} \mathbf{a}^{(0)}\right\} \equiv\left\{\mathbf{b}^{(1)} \mathbf{b}^{(0)}\right\}$, we evidently have $\left\{\mathbf{T}_{p+1}\right\} \equiv\left\{\mathbf{b}^{(1)} \mathbf{T}_{p}^{\prime}\right\}$. The remainder of the proof follows as easily and may be omitted, the statement following.

Theorem 5. The transformation $\left\{\mathbf{a}^{(1)} \mathbf{a}^{(2)} \cdots \mathbf{a}^{(r)}\right\}$ compounded of $r$ inde. pendent central reflections establishes within the $M_{r-1}$ numerically derived from the centers $a^{(1)}, a^{(2)}, \ldots a^{(r)}$, a duality which is characteristic of the transformation. We may resolve the transformation in $\infty^{\frac{1}{2} r(r-1)}$ ways, the first center being any point in $M_{r-1}$, and the following limited only by the condition that the $M_{r-2}$ corresponding to any one of them in the duality contains all the succeeding centers. Coincidence of point and corresponding $M_{r-2}$ occurs when and only when the point is on the absolute. The transformation depends upon $r(n-1)-\frac{1}{2} r(r-1)=\frac{1}{2} r(2 n-r-1)$ essential parameters.

From this result we may at once state

Theorem 6. Any product of reflections in centers lying in an $M_{r-1}$ may always be reduced to one of $r$ factors or fewer. The most general transformation compounded of central reflections may therefore be reduced to one of $n$ factors

The proof follows at once from theorem 5 . For example, $\left\{\mathbf{a}^{(1)} \mathbf{a}^{(2)} \cdots \mathbf{a}^{(n)} \mathbf{a}\right\}$ may be written $\left\{\mathbf{b}^{(1)} \mathbf{b}^{(2)} \cdots \mathbf{b}^{(n-1)} \mathbf{a} \mathbf{a}\right\}$ or $\left\{\mathbf{b}^{(1)} \mathbf{b}^{(2)} \cdots \mathbf{b}^{(n-1)}\right\}$. For any, $\mathbf{T}_{p}=\left\{\mathbf{a}^{(1)} \mathbf{a}^{(2)} \cdots \mathbf{a}^{(\mathbf{p})}\right\}$ the polar $M_{n-p-1}$ with respect to $f=0$ of the locus of centers is a locus of invariant points. All remaining invariant points lie in the locus of centers.

\section{$\S 2$. On the resolution of any orthogonal transformations into central reflections.}

The quadratic form of the preceding section is now assumed to be

$$
\phi(x)=x_{1}^{2}+x_{2}^{2}+\cdots x_{n}^{2} .
$$


Let $\{\mathbf{T}\}$, defined by the equations

$$
y_{i}=\sum_{i} \alpha_{i k} x_{k}
$$$$
(i, k=1,2, \cdots, n) \text {, }
$$

be any orthogonal transformation, i. e., $\phi(y) \equiv \phi(x)$. The question is to show that $\{\mathbf{T}\}$ is a product of central reflections. This is known to be the case for $n=2$, for example. For if we interpret $x_{1}$ and $x_{2}$ as Cartesian rectangular coordinates in the plane, $\{\mathbf{T}\}$ is either a rotation around the origin or a reflection * in a line through the origin according as the determinant $\left|\alpha_{i k}\right|$ in (4) is 1 or -1 . But in the case of a rotation, $\{\mathbf{T}\}$ is compounded of two line reflections.

Knowing, then, that the orthogonal transformation for $n=2$ is always a product of central reflection, I shall prove the theorem true in general by establishing the truth of the proposition :

An orthogonal transformation in $n$ variables may always be resolved into one in $n-1$ variables compounded with central reflections. $\dagger$

We may assume in (4) that $\alpha_{n n} \neq 0$. For if this were the case, suppose $\alpha_{n r} \neq 0$, which must be true for some value of $r$. But the reflection $\{a\}$ in the centre $a_{1}=a_{2}=\cdots=a_{r-1}=0, a_{r}=1, a_{r+1}=\cdots=a_{n-1}=0, a_{n}=-1$, merely interchanges $x_{r}$ and $x_{n}$, and, accordingly, composition of $\{\mathbf{T}\}$ and $\{a\}$ will give us a transformation for which the hypothesis holds.

Now suppose $\alpha_{n s} \neq 0$. Consider a reflection $\{\mathbf{b}\}$ for which only $b_{s}$ and $b_{r}$ do not vanish. This has the form

$$
\begin{array}{ll}
x_{k}=x_{k}^{\prime} & (k \neq s \neq n), \\
x_{s}=\lambda x_{s}^{\prime}+\mu x_{n}^{\prime}, & \\
x_{n}=\mu x_{s}^{\prime}-\lambda x_{n}^{\prime}, &
\end{array}
$$

where $\lambda^{2}+\mu^{2}=1$. Then if $\lambda: \mu$ be chosen so that $\lambda \alpha_{n x}+\mu \alpha_{n n}=0$, the compounded transformation $\{\mathrm{Tb}\}$, viz., $y_{i}=\sum_{k} \beta_{i k} x_{k}^{\prime}$, is such that $\beta_{n s}=0$, $\beta_{n n} \neq 0$, but otherwise $\beta_{n r}=\alpha_{n r}$. Hence we may multiply $\{\mathbf{T}\}$ by a product of central reflections such that finally $y_{n}=\gamma_{n n} x_{n}$. But for an orthogonal transformation $\ddagger$ we must have $\gamma_{n n}= \pm 1$, and $x_{n}$ must disappear from the other equations, and therefore the proposition is established.

Referring to the preceding theorems we may now state the fundamental result.

Theorem 7. Every linear transformation of a general quadratic form in $n$ variables into itself may be compounded of $n$ central reflections or fewer, and theorems 1-5 hold for all such transformations.

* For this interpretation of the variables, a central reflection becomes the usual reflection in a line through the origin.

† This theorem is given by KRONECKER, Berliner Monatsberichte (1890), p. 1071, whose proof is essentially identical with the following.

$\ddagger$ Since $\Sigma_{i} \alpha_{i n} \alpha_{i s}=0(i, s=1,2, \cdots, n-1)$, and also $\left|\alpha_{i n}\right| \neq 0$, we must have $\alpha_{i n}=0$, and from $\Sigma_{i} \alpha_{i n}^{2}+\gamma_{n n}^{2}=1, \gamma_{n n}= \pm 1$. 
As already remarked, Voss has proved that the general orthogonal substitution is compounded of $n$ central reflections, and for this purpose he chooses the equations of the transformation in CAYLEY's form. He also points out that the determination of the centers is exactly analogous to that of finding a selfconjugate tetrahedron with respect to a quadric.* The association of the general transformation with a characteristic duality is implied in this statement, but very imperfectly. Moreover, since the formulas of CAYLEY apply only to general proper transformations, it is evident that the proof given by Voss is incomplete.

\section{§3. Equations of the transformations $\dagger$ compounded of $n$ independent central reflections.}

Since the general transformation is completely characterized by a correlation in which coincidence of point and corresponding $M_{n-2}$ arises when the point is on the absolute, we begin with the duality $\{\Gamma\}$ defined by

$$
\sum_{i, k} \beta_{i k} x_{i} y_{k}=0 \quad(i, k=1,2, \cdots, n) .
$$

For $x=y$, this must reduce to (1), or, $f(x)=0$; i. e., $\beta_{i k}+\beta_{k i}=2 \lambda a_{i k}$. Then if we set $\beta_{i k}-\beta_{k i}=2 \mu \alpha_{i k}$, we may write $\{\Gamma\}$ in the form

$$
\sum_{i, k}\left(\lambda a_{i k}+\mu \alpha_{i k}\right) x_{i} y_{k_{i}}=0 \quad\left(a_{i k}=a_{k i}, \alpha_{i k}=-\alpha_{k i}\right) .
$$

The equation (6) gives the most general form of the correlation in question, and shows that for a given $f(x)$, the variety is given by the number of the arbitrary $\alpha$ 's, viz., $\frac{1}{2} n(n-1)$; and accordingly, the number of essential parameters in the most general Hermite iransformation is $\frac{1}{2} n(n-1)$.

The duality

$$
f(x, y) \equiv \sum a_{i k} x_{i} y_{k}=0
$$

is evidently the polar reciprocation with respect to the absolute, while

$$
w(x, y) \equiv \sum \alpha_{i k} x_{i} y_{k}=0
$$

$\left(a_{i k}=-a_{k i}\right)$

is a nullsystem, since point and corresponding $M_{n-2}$ lie in coincidence. The geometrical relation of the dualities (6), (7) and (8) is simply this. Under $\{\Gamma\}$ the point $x$ gives the two $M_{n-2}$ 's, $M$ and $M^{\prime}$ say, whose equations are $\lambda f(x, y)+\mu w(x, y)=0, \lambda f(x, y)-\mu w(x, y)=0$; and therefore $M$ and $M^{\prime}$ are divided harmonically by the polar of $x$ with respect to the absolute and the correspondent of $x$ in the nullsystem. $\ddagger$

* Loc. cit., p. 349.

$\dagger$ It will be convenient to designate any linear transformation of a quadratic form into itself, an Hermile transformation. (Cf. LOEWY, loc. cit.)

$\ddagger$ Cf. Vorlesungen über Geometrie, Clebsch-Lindemans, vol. 2, p. 40", for the corresponding discussion in ordinary space. 
We proceed now to find the equations of the Hermite transformation $\{\mathbf{T}\}$ corresponding to the correlation $\{\boldsymbol{\Gamma}\}$ defined by (6).

Let $x\{\mathbf{T}\} x^{\prime}$, and suppose $\{\mathbf{T}\} \equiv\left\{\mathbf{a}^{(1)} \mathbf{a}^{(2)} \cdots \mathbf{a}^{(n)}\right\}$, according to theorem 7 . Choose $a$ such that $a\{\Gamma\} M$, when $M$ is the polar of $x^{\prime}$, i. e.,

$$
\sum_{i}\left(\lambda a_{i k}+\mu \alpha_{i k}\right) a_{i} \equiv \rho \sum_{i} a_{i k} x_{i}^{\prime} \quad(i, k=1,2, \cdots, n) .
$$

Then $x^{\prime}$ is an invariant point for $\{\mathbf{a T}\}$, and hence $x\{a\} x^{\prime}$. That is, we have to eliminate the $a$ from (9) and

$$
x=x^{\prime}-\frac{2 f\left(x^{\prime}, a\right)}{f(a)} a .
$$

Multiplying (9) by $a_{k}$ and summing with respect to $k$, gives $\lambda f(a)=\rho f\left(x^{\prime}, a\right)$; hence (10) becomes

$$
x=x^{\prime}-\frac{2 \lambda}{\rho} a ;
$$

and now eliminating $a$ from (9) and (11) we obtain

$$
\sum_{i}\left(\lambda a_{i k}-\mu \alpha_{i k}\right) x_{i}^{\prime}+\sum_{s}\left(\lambda \alpha_{s k}+\mu \alpha_{s k}\right) x_{s}=0 \quad(i, k, s=1,2, \cdots, n) .
$$

The equations $\dagger$ of $\{\mathbf{T}\}$ are then found by solving (12) for $x^{\prime}$. Before taking up this question, however, an interesting geometrical theorem is at once read out of (12), viz.,

TheOREM 8. Corresponding points in any general Hermite transformation are points which give the same $M_{n-2}$ in the duality which is characteristic of the transformation.

This theorem completes the connection between the transformation $\{\mathbf{T}\}$ and its corresponding duality, and would serve as basis of a purely geometrical discussion.

The equations of $\{\mathbf{T}\}$ in the form $\dagger(12)$ are very convenient for discussion as the following deductions will show.

Write (12) when solved for $x^{\prime}$ in the form

$$
x_{i}^{\prime}=\sum_{k} c_{i k} x_{k} \quad(i, k=1,2, \cdots, n) .
$$

Then denoting the determinant $\left|\lambda a_{i k}+\mu \alpha_{i k}\right|$ by $\Delta(\lambda, \mu)$, we evidently have

$$
\left|c_{i k}\right| \equiv(-1)^{n} \frac{\Delta(\lambda, \mu)}{\Delta(\lambda,-\mu)}=(-1)^{n}
$$

for $\Delta(\lambda,-\mu)$ is simply $\Delta(\lambda, \mu)$ with rows and columns interchanged. This

* Multiplying (12) by $x_{k}^{\prime}$ and $x_{k}$, respectively, and summing with respect to $k$ gives $f(x)=f\left(x^{\prime}\right)$, a verification.

$\dagger$ I do not find the Hermite transformation so given elsewhere. See, for example, Loewy, loc. cit., pp. 9 et seq. 
result is a verification, for the determinant of a central reflection is -1 , and of course of $\{\mathbf{T}\}$, should be $(-1)^{n}$. Hence

Theorem 9. The equations (12) define a proper or improper Hermite transformation according as $n$ is even or odd.

The solution of (12) is obtained at once by first solving (9) for the $a$ 's and then substituting in (11). Following this method we readily find for the $c$ 's of (13) the equations :

$$
c_{i i}=\frac{\Delta(\lambda, \mu)-2 \lambda \sum_{k} a_{k i} \Delta_{k i}(\lambda, \mu)}{\Delta(\lambda, \mu)}, \quad c_{i s}=\frac{-2 \lambda \sum_{k} a_{k s} \Delta_{k i}(\lambda, \mu)}{\Delta(\lambda, \mu)}
$$

The classical formulas of Cayley for an orthogonal substitution are of course obtained by setting $a_{i k}=0, i \neq k, a_{i i}=1$.

Associated with the same nullsystem $\alpha_{i k}$ are $\infty^{1}$ dualities determined by the ratio $\lambda: \mu$. An interesting result is obtained by putting $\mu=0:$ for then the duality becomes the polar reciprocation in the absolute. The centers of $\{\mathbf{T}\}$ are now conjugate in pairs and therefore reflections in these centers are commutative $(\S 1)$. The equations (12) become simply $x_{i}^{\prime}=-x_{i}$, and we get

Theorem 10. The transformation in $R_{n-1}$ compounded of $n$ commutative central reflections in the absolute reduces to an identical point transformation.

This result is obtained by Voss (loc. cit., p. 345), who also gives interesting geometrical consequences for $n=3$ and $n=4$. Compounding $\{\mathrm{T}\}$ with $x_{i}^{\prime}=-x_{i}$ gives a proper transformation for every $n$. If $n$ is odd, however, the product reduces down to one of $n-1$ central reflections. In this case, therefore, the transformation compounded of $n$ and of $n-1$ central reflections are essentially identical.

The unsolved form (12) of $\{\mathbf{T}\}$ is* very convenient for discussion of fixed elements. For, putting $x^{\prime}=\rho x$, we find

$$
\phi(\rho) \equiv\left|\lambda a_{i k}(\rho+1)-\mu \alpha_{i k}(\rho-1)\right|=0,
$$

the corresponding fixed point $x$ being given by

$$
\sum_{i}\left[\lambda \alpha_{i k}(\rho+1)-\mu \alpha_{i k}(\rho-1)\right] x_{i}=0 .
$$

Evidently $\phi(1) \neq 0$ if $\left|a_{i k}\right| \neq 0$, and we learn that the characteristic equation $\dagger$ of the transformation (15) cannot have the root +1 if the absolute is nondegenerate.

Geometrically, the determination of the fixed elements is the same as the

* Cf., e. g., Linderann, Münchener Berichte (1896), p. 52.

$\dagger$ The "characteristic function" of (13), (15) is readily seen to be $\phi(\rho) \div(-1)^{n} \Delta(\lambda, \mu)$, since for $\mu=0$, the characteristic function must reduce to $\left|c_{i k}\right|$ or $(-1)^{n}$. 
question of coincident elements in the polar reciprocation (7) and the nullsystem (8).

\section{\$4. The transformations compounded of $n$ central reflections or fewer.}

The transformation $\left\{\mathbf{T}_{n-t}\right\} \equiv\left\{\mathbf{a}^{(1)} \mathbf{a}^{(2)} \cdots \mathbf{a}^{(n-t)}\right\}, t>0$, becomes a $\left\{\mathbf{T}_{n}\right\}$ if multiplied by any $\left\{\mathbf{T}_{t}\right\}$ whose locus of centers does not intersect the locus of centers of $\left\{\mathbf{T}_{n-t}\right\}$. The correlation within the latter $M_{n-t-1}$ which is characteristic of $\left\{\mathbf{T}_{n-t}\right\}$ is therefore determined by a duality (6), in the sense that a point in $M_{n-t-1}$ corresponds to the $M_{n-t-2}$ determined by the intersection of $M_{n-t-1}$ and the $M_{n-2}$ corresponding to the point by (6). Furthermore, it is evident that the relation

$$
\left\{\mathbf{T}_{n}\right\} \equiv\left\{\mathbf{T}_{t} \mathbf{T}_{n-t}\right\}
$$

indicated above, implies that the locus of centers of $\left\{\mathbf{T}_{t}\right\}$ corresponds under the duality defining $\left\{\mathbf{T}_{n}\right\}$ to the locus of centers $M_{n-t-1}$ of $\left\{\mathbf{T}_{n-t}\right\}$. As remarked before, these must not intersect. The necessary and sufficient condition is found. For if $M_{n-t-1}$ is defined by

$$
\left(\nu^{(1)} x\right)=0,\left(\nu^{(2)} x\right)=0, \cdots,\left(\nu^{(t)} x\right)=0,
$$

then the corresponding $M_{t-1}$ under (6) is determined from

$$
\sum_{i}\left(\lambda a_{i k}+\mu \alpha_{i k}\right) a_{i}=\sigma^{(1)} \nu_{k}^{(1)}+\sigma^{(2)} \nu_{k}^{(2)}+\cdots+\sigma^{(t)} \nu_{k}^{(t)} \quad(i, k=1,2, \cdots, n) .
$$

If then the $a$ 's satisfy (16), the condition appears in the vanishing of the determinant formed by bordering $\Delta(\lambda, \mu)$ by the $\nu$ 's. Denoting this bordered determinant by

we have

$$
\Delta^{(t)}(\lambda, \mu)
$$

THEOREM 11. The necessary and sufficient condition for the intersection of an $M_{n-t-1}(16)$ with the $M_{t-1}$ corresponding to it under any duality is found by equating to zero the determinant of order $n+t$ formed by bordering the determinant of the duality with the $\nu$ s.

In particular, $\Delta^{(1)}(\lambda, \mu)=0$ defines in the coördinates $\nu_{i}$ the correlate of the absolute itself under $\{\Gamma\}$. That is, the envelope of the $M_{n-2}$ 's which contain their corresponding points. This quadratic manifold is the second "fundamental locus" of the duality, the other being the absolute itself.*

Proceeding now to find the equations of $\left\{\mathbf{T}_{n-t}\right\}$, the locus of centers being defined by $(16)$ and $\Delta^{(t)}(\lambda, \mu) \neq 0$, we may at once write down the equations of the most general duality within $M_{n-t-1}$ such that the $M_{n-t-2}$ determined by any point shall lie in the $M_{n-2}$ corresponding to that point under (6). These are

* Lindemann, Vorlesungen etc., vol. 2 , p. 402 ; Voss, Mathematische Annalen, vol. 13 (1878), p. 359. 
$\sum_{i}\left(\lambda a_{i k}+\mu x_{i k}\right) a_{i}+\sigma^{(1)} \nu_{k}^{(1)}+\sigma^{(2)} \nu_{k_{k}}^{(2)}+\cdots+\sigma^{(t)} \nu_{k}^{(t)}=\rho u_{k} \quad(i, k=1,2, \cdots, n)$.

The $a$ 's satisfy (16) also, or

$$
\left(\nu^{(1)} a\right)=0,\left(\nu^{(2)} a\right)=0, \cdots,\left(\nu^{(t)} a\right)=0 .
$$

The $n+t$ equations (18) and (19) may be solved for the $a$ 's and $\sigma$ 's, since $\Delta^{t}(\lambda, \mu) \neq 0$. The $a$ 's depend only upon the intersection of $(u x)=0$ and the $M_{n-t-1}(16)$.

As before, if $x\left\{\mathbf{T}_{n-t}\right\} x^{\prime}$, then choosing for $u$ the polar $M_{n-2}$ of $x^{\prime}$ with respect to $f=0$, i. e., taking in (18),

$$
u_{k}=\sum_{i} a_{i k} x_{i}^{\prime},
$$

and assuming the $a$ determined by (18) as the first center of $\left\{\mathbf{T}_{n-t}\right\}$, then obviously $x\{a\} x^{\prime}$, as in the general case, $\S 3$. Multiplying (18) by $a_{k}$ and summing with respect to $k$, gives by (19) and (20) $\lambda f(a)=\rho f^{\prime}\left(x^{\prime}, a\right)$. Hence the equations of the $a$ 's are

$$
x_{i}-x_{i}^{\prime}+\frac{2 \lambda}{\rho} a_{i}=0 .
$$

The equations of $\left\{\mathbf{T}_{n-t}\right\}$ are then found by the elimination of the $a$ 's, the $u$ 's, and the $\sigma$ 's from $(18),(19),(20)$, and (21). In fact substituting from (20) and (21) in (18) and (19), gives

$$
\begin{gathered}
\sum_{i}\left(\lambda a_{i k}+\mu \alpha_{i k}\right) x_{i}+\sum_{j}\left(\lambda a_{j k}-\mu \alpha_{j k}\right) x_{j}^{\prime}-\frac{2 \lambda}{\rho} \sum_{s} \sigma^{(s)} \nu_{k i}^{(s)}=0 \\
(i, j, k=1,2, \cdots, n ; s=1,2, \cdots, t), \\
\left(\nu^{(s)} x\right)-\left(\nu^{(s)} x^{\prime}\right)=0 .
\end{gathered}
$$

Multiplying (22) by $x_{k}$ and $x_{k}^{\prime}$ respectively and summing with respect to $k$ gives as before the verification

We may therefore state

$$
f(x)=f\left(x^{\prime}\right) \text {. }
$$

Theorem 12. The equations (22) and (23) define in unsolved form a linear transformation of the quadratic form $f(x)$ into itself, and every such transfortion is given by these equations, $t$ having any value from 0 to $n-1$, and the $\alpha$ 's being elements of an arbitrary skew determinant.

The form $x_{i}^{\prime}=\sum c_{i s} x_{s}$ for the transformation is readily obtained by solving (18), (19), (20) for $a_{i}$ and substituting in (21). This gives

$$
c_{i s}=e_{i s}-\frac{2 \lambda \sum_{k} a_{k s} \Delta_{k i}^{(t)}(\lambda, \mu)}{\Delta^{(t)}(\lambda, \mu)},
$$

where

$$
e_{i s}=0(i \neq s), e_{i i}=1 \quad(i, k, s=1,2, \cdots, n) .
$$


Theonem 13. The equations (24) give the form of the coefficient of every Herinite transformation.

The determinant $\left|c_{i s}\right|=(-1)^{n-t}$. This is easily seen by making a change of variable $y_{s}=\left(\nu^{(s)} x\right), s=1,2, \ldots, t$, by which $n-t$ of the $x$ 's remain unchanged, say $x_{t+1}, \cdots, x_{n}$, it being assumed that the determinant

of the matrix

does not vanish.

$$
\left|\nu_{1}^{(1)} \nu_{2}^{(2)} \cdots \nu_{t}^{(t)}\right|
$$

This transformation may readily be effected by first eliminating the $\sigma$ 's, and then changing the variables.

The $\alpha$ 's are still arbitrary in (24), but no longer essential. In fact, (18) and (19) defining a duality in a linear $M_{n-t-1}$, contain at most $\frac{1}{2}(n-t)(n-t-1)$ essential parameters. And indeed, if the $\sigma$ 's and $a_{1}, a_{2}, \ldots, a_{t}$ be eliminated from (18) and (19) under the hypothesis (25) made above, there will remain $(n-t)$ equations for $a_{t+1}, \cdots, a_{n}$, of the form

$$
\sum_{j}\left(\lambda a_{t+j, t+k}^{\prime}+\mu a_{t+j, t+k}^{\prime}\right) a_{t+j}=\rho u_{t+k}^{\prime} \quad(j, k=1,2, \cdots, n-t),
$$

in which the $a^{\prime \prime}$ s and $\alpha^{\prime \prime}$ s are linear in the $a$ 's and $\alpha$ 's respectively, and at the same time elements of a symmetric and skew determinant respectively. The coefficients of the $a$ 's and $\alpha$ 's are quadratic in the determinants of order $t$ of the matrix (25).

Since the number of independent determinants is $t(n-t)$, we find for the total number of essential parameters in (24),

$$
\frac{1}{2}(n-t)(n-t-1)+t(n-t)=\frac{1}{2}(n-t)(n+t-1), *
$$

which agrees with the statement of Theorem 5 for $r=n-t$.

Returning to (22) and (23) for discussion of fixed elements $x^{\prime}=\rho x$, we find for the characteristic function of (24),

$$
\phi(\rho)=\frac{(-1)^{n-t}(1-\rho)^{t} \phi^{(t)}(\rho)}{\Delta^{(t)}(\lambda, \mu)}
$$

where $\phi^{(t)}(\rho)$ is the determinant $(15 a)$ of the general case bordered with the $\nu$ 's. It is easy to transform $\phi^{(t)}(\rho)$ into the determinant of order $n-t$,

$$
\phi^{(t)}(\rho)=\left|\lambda a_{t+j, t+k}^{\prime}(\rho+1)-\mu \alpha_{t+j, t+k}^{\prime}(\rho-1)\right|,
$$

in which $a^{\prime}$ and $\alpha^{\prime}$ have the same significance as above. The equations (28) and (29) give the theorem due to Voss. $\dagger$

* Cf. Lindemann, M ünchener Berichte (1896), p. 66.

† Voss, Münchener Berichte (1896), p. 14. 
The characteristic function for any Hermite transformation (24), except for the factor $(1-\rho)^{t}$, always has the form

$$
\left|\lambda a_{i k}^{\prime}(\rho+1)-\mu \alpha_{i k}^{\prime}(\rho-1)\right| \quad(i, k=1,2, \cdots, n-t),
$$

in which the $a^{\prime \prime}$ s and $\alpha^{\prime \prime}$ s are elements of a symmetric and skew determinant respectively.

Finally, (22) and (23) show that $\rho=1$ gives for the corresponding fixed point

$$
\rho \sum_{i} \lambda a_{i k} x_{i}=\sum_{s} \sigma^{(s)} \nu_{k}^{(s)}
$$

i, e., every point of the polar of the $M_{n-t-1}(16)$ with respect to the absolute is fixed. Any other fixed point lies in the $M_{n-t-1}(16)$, the discussion of their arrangement being precisely that of the general case if $n$ is replaced by $n-t$.

The problem of the determination of all linear transformations of a quadratic form into itself may therefore be regarded as completely solved in this and the preceding sections. The derivation of canonical forms for any given case is a matter of no great difficulty. The question evidently depends primarily upon the discussion of the dualities (7) and (8) of $\S 3$.

\section{§5. Further resolution of the transformation into involutory transformations.}

The inverse $\left\{\mathbf{T}_{n-t}^{-1}\right\}$ of the transformation (22), (23) is found by changing $\mu$ to $-\mu$. Hence $\left\{\mathbf{T}_{n-t}\right\}$ is involutory when and only when $\mu=0$, i. e., when $\left\{\mathbf{T}_{n-t}\right\}$ is compounded of reflections in centers conjugate with respect to the absolute. Such a transformation depends upon $t(n-t)$ essential parameters, viz., the coördinates of the locus of centers.

We now state the theorem:

TheORem 14. Every linear transformation of a quadratic form into itself may be resolved into the product of two involutory transformations.

Consider any $\left\{\mathbf{T}_{t}\right\}, t=2 r$; then we shall prove

$$
\left\{\mathbf{T}_{t}\right\} \equiv\left\{\mathbf{a}_{1}, \mathbf{a}_{2}, \cdots, \mathbf{a}_{r}\right\}\left\{\mathbf{b}_{1}, \mathbf{b}_{2}, \cdots, \mathbf{b}_{r}\right\},
$$

where the $a$ 's and also the $b$ 's are conjugate in pairs.

For simplicity of statement, take $r=2$. Then the locus of centers of $\left\{\mathbf{T}_{4}\right\}$ is ordinary space, $M_{3}$, the transformation being characterized by a duality $\{\Gamma\}$ in $M_{3}$, which is pointed out in $\S 3$, is defined by means of a nullsystem $\{\mathbf{N}\}$ together with reciprocation in the absolute. From (6) and (8) $\S 3$, it is readily seen that $\left\{a_{1} a_{2}\right\} \equiv\left\{a_{2} a_{1}\right\}$ when and only when $a_{2}$ lies in the plane corresponding to $a_{1}$ under $\{\mathbf{N}\}$. Thus the line $A$ joining $a_{1}, a_{2}$ is invariant under $\{\mathbf{N}\}$, that is, $A$ "belongs to $\{\mathbf{N}\}$," and the line $B$ determined by $b_{1}, b_{2}$ enjoys the 
same property.* Furthermore $A\{\Gamma\} B$, by theorem 5. Hence, since the $\infty^{3}$ lines of $\{\mathbf{N}\}$ transform by $\{\boldsymbol{\Gamma}\}$ into the $\infty^{3}$ lines of a nullsystem $\left\{\mathbf{N}^{\prime}\right\}$, it appears that the line $A$ of $\{\mathbf{N}\}$ becomes $B$ of $\left\{\mathbf{N}^{\prime}\right\}$, that is, $B$ belongs to $\{\mathbf{N}\}$ and $\left\{\mathbf{N}^{\prime}\right\}$. Through any point $b_{1}$ passes one line of the congruence common to $\{\mathbf{N}\}$ and $\left\{\mathbf{N}^{\prime}\right\}$, hence if $b_{1}$ is chosen arbitrarily, $B$ is determined uniquely, and also $A$ from $A\{\Gamma\} B$, but $a_{1}$ is any point on $A$. Thus the resolution may be effected in $\infty^{2}$ ways.

The proof for $r>2$ is precisely the same, and the degree of freedom is found to be $r$. For $t=2 r-1$, it is only necessary to multiply $\left\{\mathbf{T}_{t}\right\}$ by an $\{\mathbf{a}\}$, and then apply the theorem, remembering that $a$ may be chosen arbitrarily. For $n=2 r-1$, it has already been remarked that the transformation is not different from $t=2 r-2$.

As a general theorem, it may be stated that the resolution $\dagger$

$$
\left\{\mathbf{T}_{t}\right\} \equiv\left\{\mathbf{T}_{\lambda} \mathbf{T}_{\mu} \mathbf{T}_{\nu} \cdots\right\}
$$

into involutory transformations may be effected in

ways.

$$
\frac{1}{2} t(t+1)-\left(\lambda^{2}+\mu^{2}+\nu^{2}+\cdots\right)
$$

\section{§6. Application to the case $n=6$.}

Special interest attaches to the case $n=6$, for then the $x$ 's satisfying $f(x)=0,\left|a_{i k}\right| \neq 0$, may be assumed as line coördinates in ordinary space, $R_{3}$, central reflection becomes the transformation defined by a nullsystem in $R_{3}$, or, inversion in a linear line complex, while the transformations $\left\{\mathbf{T}_{t}\right\}$ in question are the collineations and correlations of projective geometry, according as $t$ is even or odd. In other words, we are concerned with line geometry in the sense of PlüCKER. The involutory transformations $\left\{\mathbf{T}_{2}\right\}$ and $\left\{\mathbf{T}_{3}\right\}$ are respectively a skew reflection $\ddagger$ and polar reciprocation in a quadric. Theorem 14 now gives the well known results that a general collineation is compounded of two polar reciprocations, and the general correlation of a skew reflection and a polar reciprocation. Resolving

$$
\left\{\mathbf{T}_{6}\right\} \equiv\left\{\mathbf{T}_{2}\right\}\left\{\mathbf{T}_{2}^{\prime}\right\}\left\{\mathbf{T}_{2}^{\prime \prime}\right\},
$$

gives the theorem due to WILson, that the general collineation is compounded of three skew reflections. The resolution possesses nine degrees of freedom, and the discussion brings out some essential facts not given in the theorem of WILson.

Let $\left\{\mathbf{T}_{2}\right\}$ in (31) be $\{a b\}$. Then if $x$ and $x^{\prime}$ are the directrices of $\left\{\mathbf{T}_{2}\right\}$,

\footnotetext{
* For discussion of the nullsystem of Möbius, reference may be made to Lindemans, Vorlesungen, vol. 2, p. 52.

† Voss has given theorem 14 for $t=n=2 r$; Mathematische Annalen, vol. 13, p. 343.

$\ddagger$ Cf. WrLson, Transactions, vol. 1 (1900), pp. 193-196.
} 
$a=x+\lambda x^{\prime}, b=x-\lambda x^{\prime}$, and from the duality $\lambda f(x, y)+\mu w(x, y)=0$ defining $\left\{\mathbf{T}_{6}\right\}$, since $a$ and $b$ satisfy this, and also $f(a, b)=0$, we find

$$
\sum \alpha_{i k} x_{i} x_{k}^{\prime}=0
$$

$\left(a_{i k}=-a_{k i}\right)$,

and the directrices of each component of $\left\{\mathbf{T}_{6}\right\}$ satisfy (32). Furthermore, it is readily found that the directrices of $\left\{\mathbf{T}_{2}^{\prime}\right\}$ and $\left\{\mathbf{T}_{2}^{\prime \prime}\right\}$ belong to the congruence common to the complexes,

$$
\lambda f(x, y)+\mu w(x, y)=0, \quad \lambda f\left(x^{\prime}, y\right)+\mu w\left(x^{\prime}, y\right)=0,
$$

and one line of this congruence may be chosen arbitrarily. The complete result therefore is

Theorem 15. A general collineation may be resolved in $\infty^{9}$ ways into the product of three skew reflections. The first pair of directrices satisfy (32) in three-dimensional space. Either one having been chosen, the other belongs to a linear line complex containing the first. The remaining four directrices then belong to the linear line congruence (33), and one having been chosen arbitrarily the other three are determined uniquely.

\section{§7. Linear transformation of the alternating bilinear form into itself.}

The alternating form

$$
w(x, y) \equiv \sum \alpha_{i k} x_{i} y_{k}=0 \quad\left(a_{i k}=-a_{k i}\right),
$$

is invariant under the transformation obtained from (22) and (23) by changing the sign of the $x^{\prime}$ in $(22)$; viz.,

$$
\begin{array}{rr}
\sum_{k}\left(\lambda a_{i k}+\mu \alpha_{i k}\right) x_{i}-\sum_{i}\left(\lambda a_{i k}-\mu \alpha_{i k}\right) x_{i}^{\prime}-\frac{2 \lambda}{\rho} \sum_{s} \sigma^{(s)} \gamma_{k}^{(s)}=0, \\
\left(\nu^{(s)} x\right)-\left(\nu^{(s)} x^{\prime}\right)=0 & (s=1,2, \cdots, t), \\
a_{i k}=a_{k i} & (i, k=1,2, \cdots, n),
\end{array}
$$

$x$ and $y$ being cogredient variables. For multiplying (35) by $y_{k}$ and summing up, with respect to $k$,

$$
\lambda f(x, y)+\mu w(x, y)-\lambda f\left(x^{\prime}, y\right)+\mu w\left(x^{\prime}, y\right)-\frac{2 \lambda}{\rho} \sum \sigma^{(s)}\left(\nu^{(s)} y\right)=0 .
$$

In the same way are found three other equations, and from these is found by using (36),

$$
w(x, y)=w\left(x^{\prime}, y^{\prime}\right) \text {. }
$$

The determinant of the solution of (35), (36) is +1 , the reasoning being the same as in $\S 4$. The characteristic function is therefore 


$$
\phi(\rho)=\frac{(1-\rho)^{t} \phi^{t}(-\rho)}{\Delta^{(t)}(\lambda, \mu)},
$$

$\phi^{(t)}(\rho)$ having same meaning as in (28).

The $a$ 's in (35) are arbitrary, but not essential parameters. The number of the latter is readily found to be

$$
\frac{1}{2}(n-t)(n-t+1)+t(n-t)=\frac{1}{2}(n-t)(n+t+1) .
$$

By the method of this section all linear transformations of the required type are found.*

*Cf. Voss, Münchener Berichte (1896), p. 20.

The Sheffikld Scientific School,

YALE UNIVHESITY. 